\title{
Notochord grafts do not suppress formation of neural crest cells or
} \section{commissural neurons}

\author{
KRISTIN B. ARTINGER and MARIANNE BRONNER-FRASER* \\ Developmental Biology Center, University of California at Irvine, Irvine, CA 92717, USA \\ *Author for correspondence
}

\section{Summary}

Grafting experiments previously have established that the notochord affects dorsoventral polarity of the neural tube by inducing the formation of ventral structures such as motor neurons and the floor plate. Here, we examine if the notochord inhibits formation of dorsal structures by grafting a notochord within or adjacent to the dorsal neural tube prior to or shortly after tube closure. In all cases, neural crest cells emigrated from the neural tube adjacent to the ectopic notochord. When analyzed at stages after ganglion formation, the dorsal root ganglia appeared reduced in size and shifted in position in embryos receiving grafts. Another dorsal cell type, commissural neurons, identified by CRABP and neurofilament immunoreactivity, differentiated in the vicinity of the ectopic notochord. Numerous neuronal cell bodies and axonal processes were observed within the induced, but not endogenous, floor plate 1 to 2 days after implantation but appeared to be cleared with time. These results suggest that dorsally implanted notochords cannot prevent the formation of neural crest cells or commissural neurons, but can alter the size and position of neural crest-derived dorsal root ganglia.

Key words: neural crest cells, dorsal root ganglion, commissural neurons, ventral roots.

\section{Introduction}

The central nervous system arises from the neural tube, which enlarges in the head region to form the brain and forms the spinal cord in the trunk. The peripheral nervous system arises from the neural crest, a population of cells originating within the dorsal neural tube. The neural tube begins as a pseudostratified columnar epithelium composed of apparently homogeneous precursor cells that later differentiate into numerous types of neurons and glia. Soon after its formation, the neural tube begins to display a characteristic polarity along the dorsoventral axis. From the dorsal aspect, neural crest cells emerge, migrate extensively and give rise to numerous and varied cell types. In the trunk, neural crest cells travel to their final destinations along two routes: (1) a ventral pathway through the rostral half of each somitic sclerotome (Rickmann et al., 1985; Bronner-Fraser, 1986) and (2) a dorsolateral pathway underneath the ectoderm (LeDouarin, 1974; Serbedzija et al., 1989). Neural crest cells that migrate along the ventral pathway differentiate into neurons and glia of the sensory and sympathetic ganglia, adrenomedullary cells, Schwann cells and chromaffin cells; those cells that migrate along the dorsolateral pathway give rise to melanocytes. Although their migratory paths are well understood, virtually nothing is known about the factors that influence formation of the neural crest and their emigration from the neural tube. Even after neural tube closure, a common precursor for neural tube and neural crest cells exists in the dorsum of the neural tube, suggesting that the neural crest is not a presegregated population (Bronner-Fraser and Fraser, 1988, 1989). Thus, the fates of neural crest and dorsal neural tube cells appear to be intimately interlinked. Other dorsal neural tube cells differentiate, in the midline, into roof plate cells and, laterally, into commissural neurons which project their axons ventrally. The ventral neural tube develops a floor plate in the midline and pools of motor neurons ventrolaterally.

The polarity of the neural tube may, in part, be set-up by extrinsic factors. For example, grafting and ablation experiments suggest that the notochord confers ventral properties on the neural tube. A grafted notochord can induce the formation of motor neurons as well as a floor plate, recognizable by its characteristic wedge-shaped cell morphology (van Straaten et al., 1988, 1989; Smith and Schoenwolf, 1989) and expression of floor-plate-specific markers (Yamada et al., 1991). In young avian embryos, any portion of the neural tube, including the dorsal-most region, is competent to form floor plate cells and motor neurons when juxtaposed to the notochord (Yamada et al., 1991).

Is the notochord sufficient to generate dorsoventral differences in the neural tube? If so, the notochord may inhibit 
or subvert formation of dorsal structures such as neural crest cells and commissural neurons. A notochordal influence on neural crest cells may not be unexpected, since it appears to inhibit ventrally migrating neural crest cells in its immediate vicinity (Newgreen et al., 1986; Pettway et al., 1990). Here, we examine whether the notochord alone is sufficient to polarize the neural tube along the dorsoventral axis. A notochord was grafted within or near the dorsal neural tube prior to neural crest cell emigration to examine if it would suppress formation of neural crest cells and/or commissural neurons. The results show that neural crest cells and commissural neurons differentiate even in the presence of a grafted notochord. However, the notochord does alter the size and position of some neural crest derivatives.

\section{Materials and methods}

\section{Preparation of notochord for implantation}

Quail embryos (Coturnix coturnix japonica) were incubated at $38^{\circ} \mathrm{C}$ until the embryos reached stage 12-13 (Hamburger and Hamilton, 1951). The trunk region was dissected out of the embryo using an electrolytically sharpened tungsten needle. The notochords were isolated from surrounding tissues with collagenase (160 units/ml; Worthington Biochemical, Freehold, NJ) for 15 minutes on ice, followed by 7 minutes at $37^{\circ} \mathrm{C}$. The notochords then were allowed to recover in complete medium consisting of Modified Eagle's Medium (MEM) plus 15\% horse serum and 10\% embryo extract for one hour on ice. For control experiments, the notochords were isolated in the same manner and then fixed in $4 \%$ paraformaldehyde in $0.1 \mathrm{M}$ phosphate buffer (PB) for 2 hours. Prior to implantation, the fixed notochords were rinsed twice with complete medium where they remained for approximately 1 hour.

\section{Preparation of chick hosts}

White Leghorn chicken embryos were incubated at $38^{\circ} \mathrm{C}$ for approximately 36 hours by which time they reached stage 9-11 (Hamburger and Hamilton, 1951). The eggs were washed with $70 \%$ ethanol, $1.5 \mathrm{ml}$ of albumen was removed, a window was cut in the shell over the embryo and India ink (Pelikan Fount, Hanover, FGR) diluted 1:4 in Howard Ringer's solution was injected under the blastoderm to aid in visualization of the embryo. The vitelline membrane was deflected using an electrolytically sharpened tungsten needle. Following the surgery, the egg was sealed with cellophane tape (Scotch Magic 3M) and returned to the incubator until the time of fixation.

\section{Microsurgery}

The types of microsurgical operations are summarized diagrammatically in Fig. 1. In order to place a notochord in the dorsalmost portion of the neural tube, it was placed in the midline of the closing neural tube prior to fusion of the neural folds (Fig. 1A). This operation often prevented the neural folds from fusing, resulting in the induction of a floor plate in the dorsal midline where the roof plate would normally form (Yamada et al., 1991); in other cases, the neural tube closed, resulting in a notochord over the dorsal-most aspect of the neural tube. For notochord grafts lateral to the neural tube, a pulled glass needle was used to make an incision 3-5 somites in length in the unsegmented mesoderm region lateral to the neural tube. A donor notochord was transferred in $2 \mu \mathrm{l}$ of medium using a pipetman and was placed in the vicinity of the incision. The notochord was oriented parallel to the incision and inserted between the dorsal neural tube and
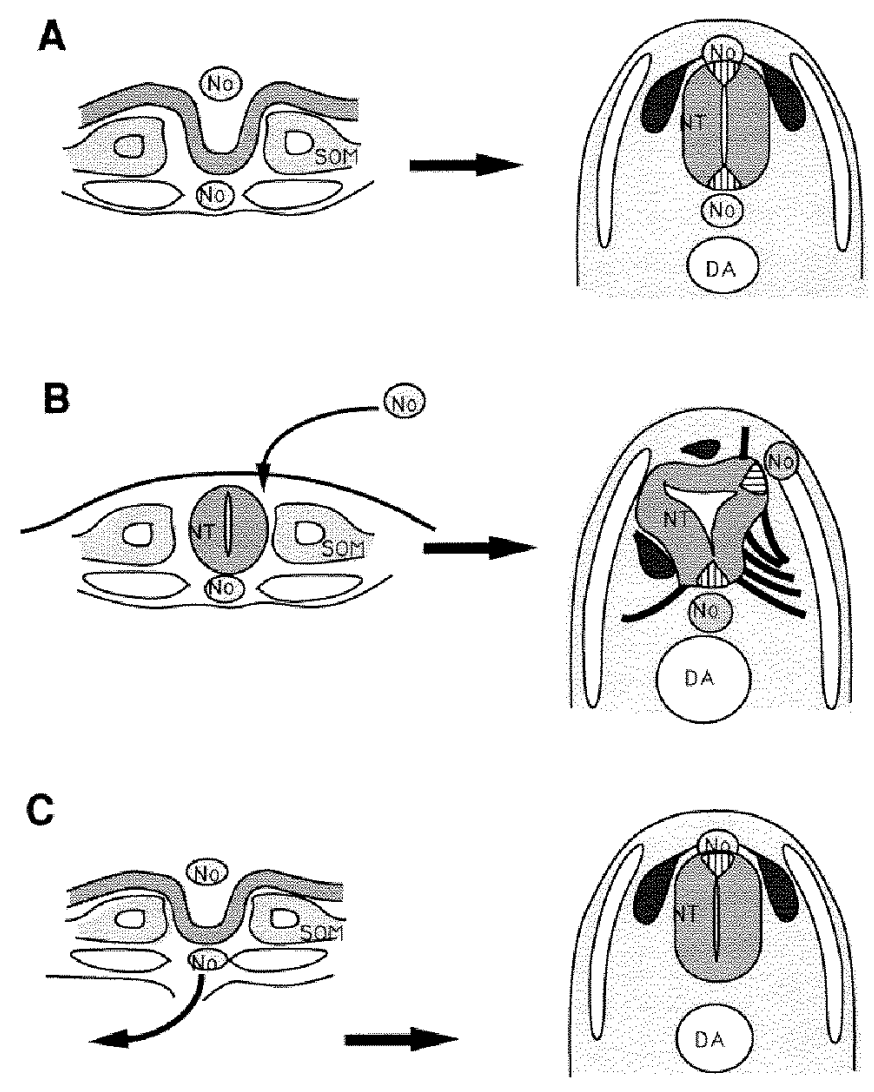

Fig. 1. Schematic diagram illustrating the microsurgical operations used in this study. (A) Implantation of an ectopic notochord in the dorsal midline prior to neural tube closure. (B) Implantation of an ectopic notochord lateral to the dorsal neural tube after tube closure. (C) Implantation of an ectopic notochord as in (A), with the host notochord removed.

the unsegmented mesoderm using a glass needle (Fig. 1B). The embryos were allowed to survive for 1-3 days after the operation.

In order to ablate the notochord, an incision was made along both sides of the neural tube of stage $9-10^{+}$embryos. A third incision was made perpendicular and caudal to the first incisions and the neural plate was carefully lifted and folded forward. The notochord was scraped off the underside of the neural plate in the posterior region of the embryo. Approximately 300-400 $\mu \mathrm{m}$ worth of notochordal tissue was removed and the neural plate was returned to its original position; this resulted in the absence of the notochord over 5-10 somite lengths in the embryo. Following removal of the endogenous notochord, another notochord was grafted into a dorsal position in the same embryo as described above (Fig. 1C).

\section{Focal injections of DiI prior to notochord implantation}

Neural fold tissue was labelled by a unilateral, focal injection of DiI (1,1-dioctadecyl-3,3,3',3'-tetramethylindocarbo-cyanine perchlorate; Molecular Probes) as described previously (Serbedzija et al., 1989, 1990). A $0.1 \%$ solution of DiI was made from an $0.5 \%$ stock solution in $100 \%$ ethanol diluted 1:5 in 0.3 M sucrose. The injection micropipettes were back filled with the DiI solution and attached to a pressure injection apparatus. Then the micropipette was inserted into a small region of the neural fold on one side of the embryo using a micromanipulator (Narashige) and a small amount of the DiI solution was expelled. Residual DiI crystals were washed away with Ringer's solution. Then, a noto- 
chord was placed into the closing neural plate as described above. The egg was sealed with adhesive tape and returned to the incubator. Embryos were fixed overnight in $4 \%$ paraformaldehyde $/ 0.25 \%$ glutaraldehyde at $4^{\circ} \mathrm{C}$, rinsed and incubated in phosphate-buffered saline (PBS) for 2-3 hours, placed in 5\% sucrose in PBS containing $0.01 \%$ azide for $2-4$ hours and into $15 \%$ sucrose in PBS at $4{ }^{\circ} \mathrm{C}$ overnight. The tissue was embedded in $7.5 \%$ gelatin (Sigma; $300 \mathrm{Bloom}$ ) in $15 \%$ sucrose/PBS at $37^{\circ} \mathrm{C}$ for $2-3$ hours, oriented and frozen in Tissue Tek OCT (Miles Laboratory) in liquid nitrogen. $10-14 \mu \mathrm{m}$ frozen sections were cut on a Zeiss Microm cryostat (Carl Zeiss Instruments) and observed the same day.

\section{Immunohistochemistry}

\section{Neurofilament antibody}

For identifying neurons and their processes, embryos were stained with an antibody against a nonphosphorylated epitope of rat neurofilament provided by Dr Virginia Lee (Lee et al., 1987). Embryos were fixed overnight in methanol at $4^{\circ} \mathrm{C}$. They were then prepared for cryostat sectioning by washing in $0.1 \mathrm{M} \mathrm{PB}$ for 1 hour followed by $5 \%$ sucrose in $0.1 \mathrm{M}$ PB overnight. Embryos were placed into $15 \%$ sucrose overnight at $4{ }^{\circ} \mathrm{C}$, embedded in $15 \%$ sucrose $/ 7.5 \%$ gelatin (Sigma), rapidly frozen in liquid nitrogen and serially sectioned at a thickness of $10 \mu \mathrm{m}$ on a cryostat (Zeiss Microm). Sections were mounted on gelatin-subbed slides. Slides were stained with the neurofilament antibody (diluted 1:300 in $0.1 \%$ BSA in PBS) overnight at room temperature. After washing in PBS, sections were stained with a highly fluorescent FITCconjugated goat anti-mouse IgG (Antibodies, Inc.) for 1 hour. Slides were coverslipped with glycerol $(90 \%$ glycerol/10\% $0.5 \mathrm{M}$ sodium carbonate, $\mathrm{pH} \mathrm{7.8,} \mathrm{containing} 2 \mathrm{mg} / \mathrm{ml}$ 1,4-diazabicyclo(2,2,2,)octane and viewed on an Olympus Vanox epifluorescence microscope. Following observation, the coverslip was removed from some of the neurofilament stained sections and they were restained with $\mathrm{HNK}-1$ or SC-1 antibodies as described below.

\section{HNK-1 antibody}

In most cases, embryos were fixed for 1 hour in Zenker's fixative and then prepared for paraffin embedding. Embryos to be embedded in paraffin were dehydrated through a graded series of ethanols, followed by three washes in Histosol (National Diagnostics), three changes of paraplast, then embedded in fresh paraplast. Blocks were sectioned on a Leitz microtome at $10 \mu \mathrm{m}$ thickness and placed on slides coated with albumin. The sections were deparaffinized in two changes of histosol, followed by rehydration in $100 \%, 100 \%, 90 \%, 70 \%$ and $30 \%$ ethanol for 1 minute each. After the final ethanol step, slides were rinsed for one minute in $0.1 \mathrm{M}$ PB. Slides were incubated with HNK-1 antibody (hybridoma supernatant) for 2 hours at room temperature or overnight at $4^{\circ} \mathrm{C}$. After rinsing with $0.1 \mathrm{M} \mathrm{PB}$ for 3 minutes, the sections were incubated with TRITC-conjugated goat anti-mouse IgM (Zymed) for one hour, washed and coverslipped as described above. For analysis of dorsal root ganglion size in HNK-1 immunostained embryos, the area of the ganglia was measured on the operated and unoperated sides of the embryo as described previously (Bronner-Fraser, 1985).

\section{SC-1 antibody}

Embryos were fixed in $4 \%$ paraformaldehyde or as described above for neurofilament immunoreactivity and cryostat sectioned. SC-1 antibody (kindly provided by Drs $H$. Tanaka and T. Yamada), which recognizes floor plate and motor neurons, was applied overnight to some slides. After rinsing, sections were incubated with an FITC-conjugated goat anti-mouse IgG (Zymed) for one hour. The secondary antibodies were diluted $1: 60$ in $0.1 \%$
BSA in phosphate-buffered saline (PBS), washed and coverslipped as described above.

\section{CRABP antibody}

An antibody against cellular retinoic acid binding protein (CRABP; kindly provided by Dr Ulf Eriksson; Eriksson et al., 1987) was used to recognize commissural neurons (Maden et al., 1989). Embryos were fixed in $20 \%$ isopropanol, $4 \%$ paraformaldehyde and $2 \%$ TCA overnight at $4{ }^{\circ} \mathrm{C}$, embedded in paraffin and serially sectioned. Slides were dewaxed, rehydrated through a graded series of ethanols and placed in PBS. They were then blocked with goat serum (diluted 1:100 in PBS) for 40 minutes. Slides were incubated with an antibody against CRABP (diluted 1:1000) overnight at $4^{\circ} \mathrm{C}$. After three washes in PBS, slides were stained with an $\mathrm{ABC}$ Elite kit (Vector laboratories) containing a horseradish-peroxidase-conjugated anti-rabbit IgG secondary antibody in order to visualize the CRABP antibody. Briefly, the secondary antibody was added for 40 minutes at room temperature, the slides were washed three times in PBS, and diaminobenzidine ( $5 \mu \mathrm{g} / \mathrm{ml}$ of Tris buffer) was added for 5-10 minutes. The slides were washed again in PBS, counterstained, dehydrated and mounted in Permount. Slides were viewed on a Vanox Microscope equipped with differential interference contrast optics.

\section{Results}

\section{Effects of a dorsal notochord implant on emigration of HNK-1 immunoreactive neural crest cells}

A notochord was placed either within, on top of or lateral to the dorsal neural tube in 10 host embryos. Some grafts $(n=5)$ were performed prior to neural tube closure in order to position the notochord on the dorsal-most aspect of the neural tube (Fig. 1A). In four cases, the notochord prevented fusion of the neural folds, resulting in a notochord wedged within the dorsal neural tube; this type of operation produces a floor plate in the presumptive roof plate region (Yamada et al., 1991). The remaining 5 grafts were performed shortly after tube closure to position the notochord dorsolateral to the neural tube (Fig. 1B); two of the embryos received two grafts, one notochord on each side of the host neural tube. Operations were performed at stages $10-11$, which is prior to neural crest cell emigration in the trunk and when the neural tube is competent to respond to induction of ventral structures by the notochord (Yamada et al., 1991). In all 10 embryos examined at stages 17-19, the dorsal or dorsolateral portion of the neural tube assumed a wedge-shaped morphology (Fig. 2), suggesting the presence of a dorsally induced floor plate. We verified the induction of an extra floor plate in the dorsal neural tube in 3 embryos by staining with the SC1 antibody, a molecular marker for the floor plate (Yamada et al., 1991); the presence of a dorsal floor plate was similar to that described by other investigators (Yamada et al., 1991; and data not shown).

For all 10 embryos, HNK-1 immunoreactive neural crest cells emigrated from the dorsal neural tube, even when the ectopic notochord was adjacent to the dorsal midline (Fig. 2A). The HNK-1 reactive cells were observed along the surface of the neural tube (Fig. 2B), but also contacted the notochord when no other space was available. This suggests that the notochord does not prevent formation of neural crest cells or their emigration from the neural tube. 

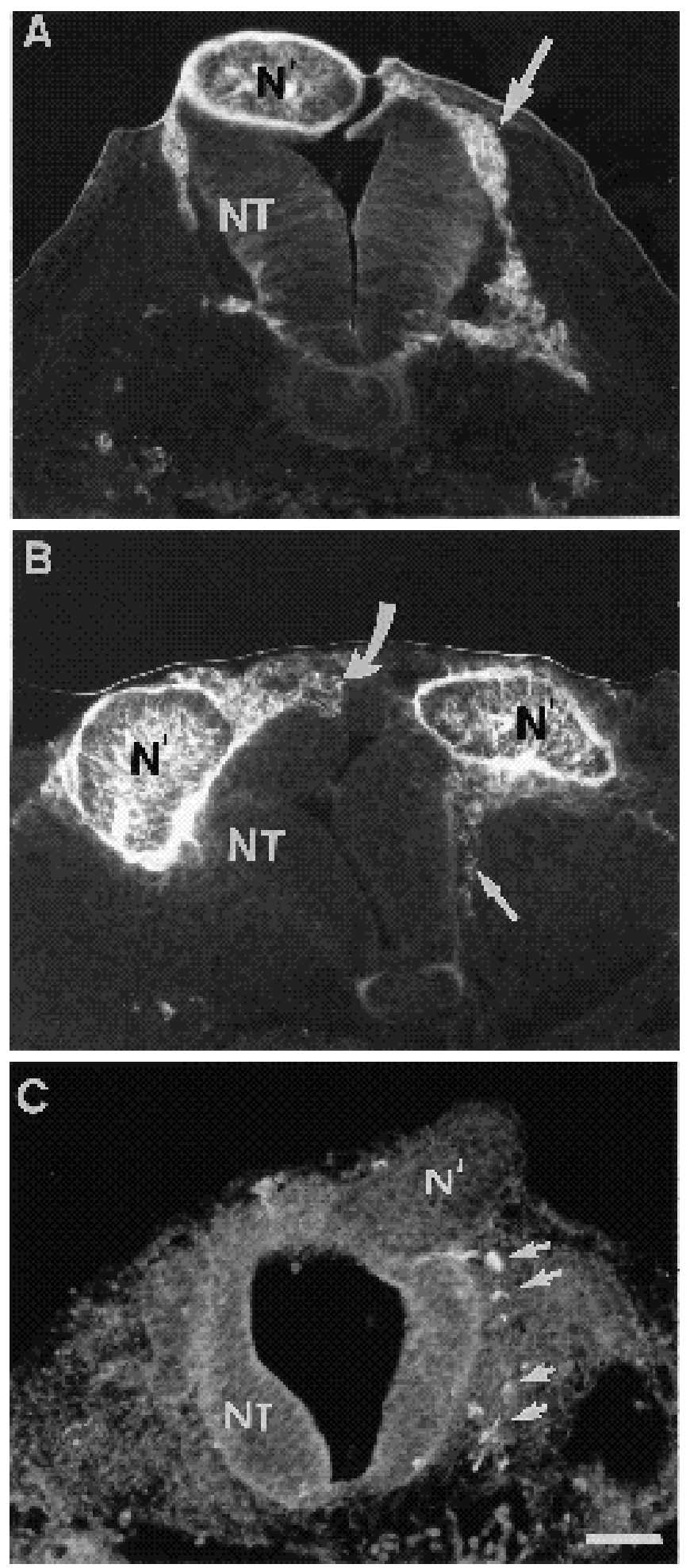

Effects of a dorsal notochord implant on emigration of DiIlabelled neural crest cells

The HNK-1 antibody is not a specific neural crest cell marker; for example, it recognizes some ventral neural tube cells (see Fig. 2A). In addition, the antibody stains neural crest cells at all axial levels, making it impossible to determine the site of origin of those HNK-1 immunoreactive cells adjacent to the implanted notochord. Thus, we cannot
Fig. 2. Fluorescence micrographs of transverse sections through embryos into which notochords were grafted adjacent to the dorsal neural tube (NT). Embryos were examined for the presence of migrating neural crest cells using the HNK-1 antibody. (A) A single ectopic notochord $\left(\mathrm{N}^{\prime}\right)$ was placed in the dorsal midline of a stage-11 embryo. When examined at stage 17 , neural crest cells (indicated by a large arrow) had emigrated from the dorsal neural tube despite the presence of the ectopic notochord. (B) An embryo in which notochords $\left(\mathrm{N}^{\prime}\right)$ were grafted on either side of the dorsal neural tube at stage 11. By stage 16, neural crest cells had emigrated from the neural tube. Some were located on the dorsal surface of the tube (curved arrow) whereas others were observed between the neural tube and somites (small arrow). (C) An embryo, fixed at stage $16+$, in which the notochord was ablated and an additional notochord was grafted to the dorsum of the neural tube at stage 10 . HNK-1 immunoreactive neural crest cells (arrows) were observed emanating from the dorsal neural tube and migrating within the rostral sclerotome. The dorsal neural tube appeared wedge-shaped in the dorsal aspect. In contrast, the ventral neural tube cells did not assume a wedge-shaped morphology (compare with ventral neural tube in $\mathrm{A}$ and $\mathrm{B}$ ). Bar = $50 \mu \mathrm{m}$ in $\mathrm{A}, \mathrm{B} ; 60 \mu \mathrm{m}$ in $\mathrm{C}$.

rule out the possibility that a dorsally implanted notochord may prevent local neural crest cell emigration, but HNK-1 reactive cells may migrate from rostral and caudal portions of the neural tube to replace the missing population. To circumvent these problems, we used an alternative approach for labelling neural crest cells by performing small focal injections of the vital dye, DiI, into the neural folds prior to neural tube closure. This makes it possible to label a small subpopulation of premigratory neural crest cells which emerges adjacent to the grafted notochord. The notochord graft was placed immediately adjacent to the labelled tissue and in some cases $(n=3)$ prevented fusion of the neural folds, becoming lodged within the dorsal neural tube.

We examined the distribution of DiI-labelled cells in 10 embryos fixed from 24 to 36 hours after implantation and labelling. 24 hours after the operation, the dorsal neural tube was clearly labelled on one side of the embryo adjacent to the ectopic notochord (Fig. 3A), but no DiI-labelled cells had emigrated from the neural tube. At a slightly later stage, DiI-labelled neural crest cells could be observed external to the neural tube, in the proximity of the neural tube and the implanted notochord (Fig. 3B). By 36 hours after operation, large numbers of DiI-labelled neural crest cells were observed within the rostral sclerotome and around the dorsal aorta (Fig. 3C,D). The pattern of DiI-labelled cells was analogous to the distribution of HNK-1 immunoreactive cells after implantation of a dorsal notochord. These results suggest that the neural crest cells can originate from the neural tube adjacent to an implanted notochord and confirm that DiI and HNK-1 labelling techniques yield similar results.

\section{Effects of a dorsal notochord implant on neural crest cell emigration in the absence of the host notochord}

In 11 embryos, the host notochord was removed (Fig. 1C) prior to implantation of a donor notochord onto the dorsal neural tube at stages $9-10^{+}$. Removing the notochord at this stage prevents formation of a morphologically detectable floor plate and motor neurons in the ventral neural tube (van 
Straaten et al., 1988, 1989; Smith and Schoenwolf, 1989; Yamada et al., 1991). After implantation of a dorsal notochord, a morphologically recognizable floor plate was detected by the wedge-shaped group of cells in the dorsal, but not ventral, portion of neural tubes in these embryos. We examined the pattern of neural crest cell emigration to determine whether neural crest cells would originate from the dorsal neural tube after induction of a dorsal 'floor plate' in the absence of the endogenous floor plate. In all embryos, HNK-1 immunoreactive neural crest cells emigrated from the dorsum of the neural tube (Fig. 2C). This demonstrates that removal of the host notochord and grafting of a dorsal notochord is not sufficient to invert the dorsoventral polarity of the neural tube.

\section{Effects of the implanted notochord on the formation of neural crest cell derivatives and ventral roots}

Neural crest cells were previously shown to avoid the space around both endogenous and implanted notochords (Pettway et al., 1990). We have extended these findings to examine the effects of the notochord on later stages of neural crest development and ganglion formation. The pattern of the peripheral ganglia was examined in 39 embryos receiving donor notochords (with or without removal of the endogenous notochord) after stage 20 , by which time neural crest-derived dorsal root ganglia have formed (Lallier and Bronner-Fraser, 1988). All of these embryos had neural tubes that appeared to have wedge-shaped protrusions adjacent to the implanted notochord; definitive floor plates could be recognized in 21 of these embryos (see Fig. 4). When the grafts were located laterally, the dorsal root ganglia were displaced to the space between the dorsal neural tube and implanted notochord, whereas the contralateral side appeared normal (Fig. 4A,B). When the notochord was implanted either at the dorsal midline or dorsolaterally, the dorsal root ganglia were displaced ventrally (Fig. 4C). In addition, the ganglia were reduced in size by $47 \pm 18 \%$ (range from 16 to $62 \%$ reduction) on the implanted side compared with the unoperated side $(n=7)$. In contrast, the sympathetic ganglia appeared to be of comparable size on both sides of the embryos. In agreement with previous findings (van Straaten et al., 1988; Stern et al., 1991; Yamada et al., 1991), an increased number of axonal bundles

Fig. 4. Fluorescence micrographs of transverse sections through embryos receiving dorsal notochord implants at stage 10-11 and examined after formation of dorsal root ganglia at stage 20-21. Embryos were stained with the HNK-1 antibody. (A) An embryo in which the implanted notochord caused a marked wedging in the neural tube (large arrow), indicating the presence of an extra floor plate. The dorsal root ganglion (D) on the implanted side was lateral to the neural tube. The ganglion was reduced in size compared with the contralateral side and was observed in a dorsal position. (B) Another embryo in which the laterally grafted notochord caused less severe 'wedging' of the neural tube.

However, the dorsal root ganglion was again displaced in position and reduced in size. (C) An embryo in which the grafted notochord was located both dorsally and laterally. The neural tube appeared wedge-shaped toward the graft and the HNK-1 immunoreactive dorsal root ganglion appeared reduced in size and displaced ventrally. Bar $=50 \mu \mathrm{m}$. emanated from the neural tube adjacent to the area of the induced floor plate (Fig. 5A,B).

\section{Effects of a fixed dorsal notochord implant on neural crest cell emigration and differentiation}

In order to determine whether a viable notochord was necessary to affect the pattern of neural crest cell emigration
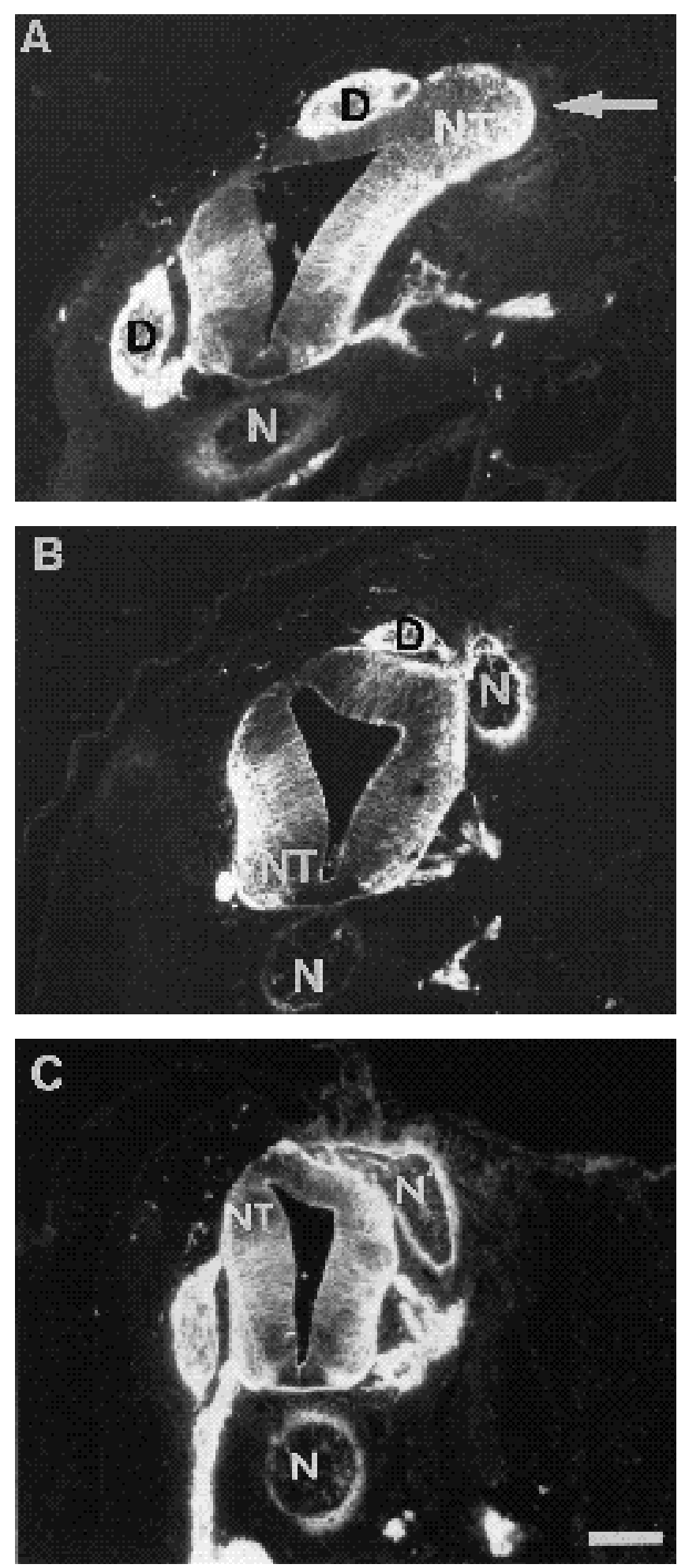

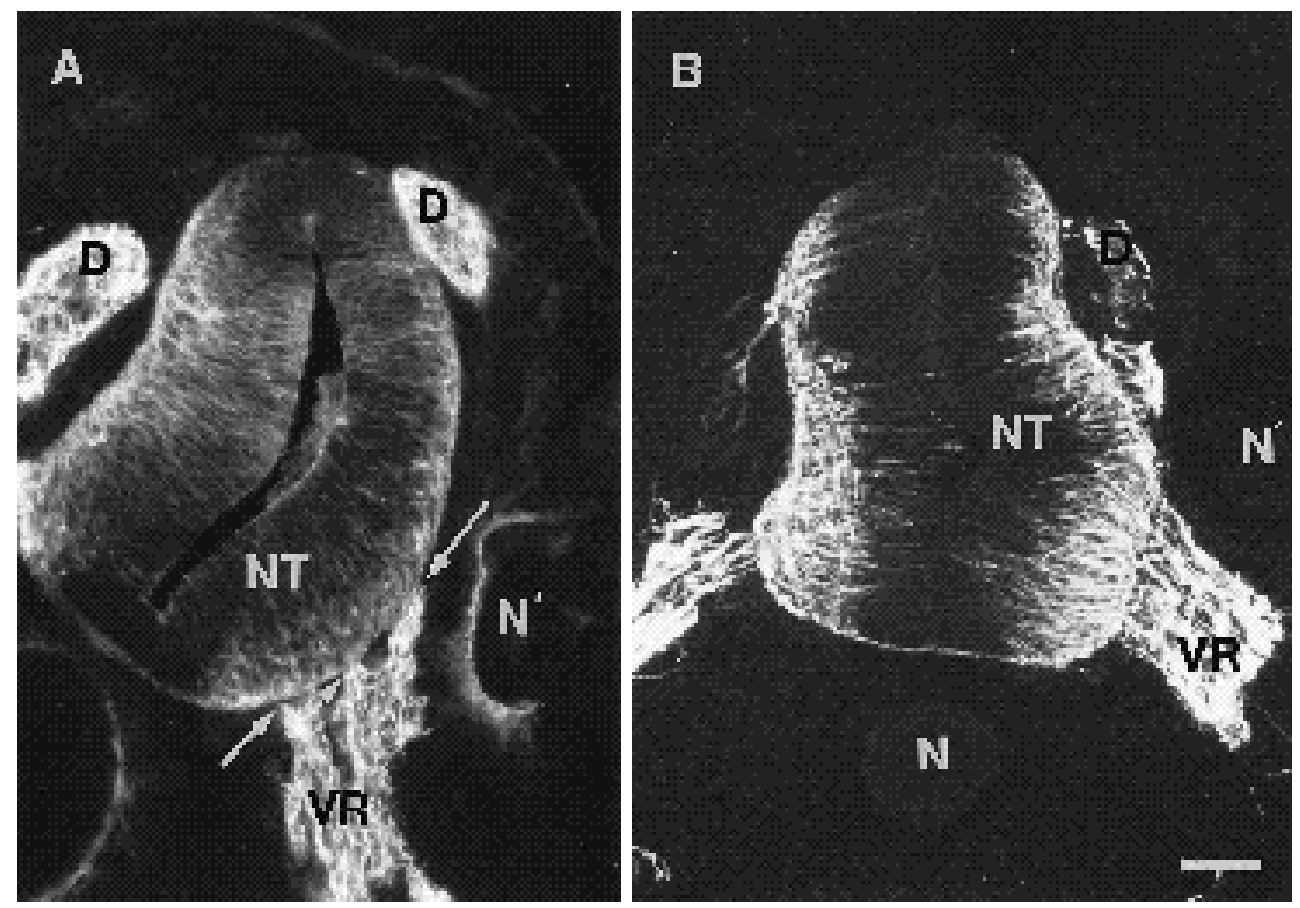

Fig. 5. Fluorescence

micrographs of transverse sections illustrating the effects of a grafted notochord on formation of the ventral roots (VR). (A) An embryo, stained with the HNK-1 antibody, in which the graft was performed at stage 11 and the embryo was examined at stage 20 . The ectopic notochord caused formation of smaller dorsal root ganglia (D) and a ventral root consisting of three bundles (arrows) of HNK-1 immunoreactive cells and axons. (B) An embryo, stained with an antibody against neurofilament. The graft was performed at stage 10 and the embryos was examined at stage 20. The width and number of neurofilament immunoreactive bundles was much greater on the grafted right side than on the unoperated side. Bar $=20 \mu \mathrm{m}$.

and differentiation, a fixed notochord was placed either lateral to or on top of the dorsal neural tube. Operations were performed at stages 9-10 and fixed at stages 17-23, during neural crest cell emigration and after ganglion formation. In all 7 embryos, no floor plate was induced by a fixed notochord. Furthermore, the position and size of the dorsal root ganglia were not significantly different on the operated versus unoperated side. This demonstrates that a live notochord is necessary to effect the pattern of neural crest development.

\section{Patterns of neuronal differentiation in the neural tube after implantation of a dorsal notochord}

Early differentiating neuronal cell bodies and axons within the neural tube can be readily identified using an antibody against neurofilament proteins. The endogenous floor plate has a characteristic pattern of neurofilament immunoreactivity, being devoid of neuronal cell bodies at most stages, but having axonal processes traversing the basal processes of its cells. Interestingly, occasional neuronal cells bodies can be observed in the endogenous floor plate region of stage 15 embryos (unpublished observation), but not at later stages. In embryos receiving dorsal or dorsolateral notochord implants, neuronal cells bodies differentiated on both sides of the neural tube. Neurofilament-immunoreactive neurons with rounded cell bodies and ventrally directed axons, characteristic of commissural neurons, were observed in the induced floor plate region of embryos fixed at stages $15-19(n=10$; Fig. 6A-D). In addition to neuronal cell bodies, numerous axonal processes were observed within the induced floor plate region. This contrasts with the endogenous floor plate, which lacked neuronal cells bodies or processes at these stages. In embryos fixed 3 days after implantation (stages 22-24; $n=5$ ), neuronal cell bodies were no longer observed in the induced floor plate region.
Instead, axonal processes were seen near the basal surface, similar to those noted in the endogenous floor plate region (Fig. 6E,F). These results suggest that the neurons are removed from the induced floor plate region, either by displacement or selective cell death after their initial differentiation. Thus, the endogenous and induced floor plates appear different with respect to neurofilament immunoreactivity 1 and 2 days post-implantation, but similar by 3 days. Because neuronal cell bodies are observed occasionally within the endogenous floor plate of young embryos (unpublished observation), this distinction may represent a temporal rather than phenotypic difference.

\section{Effects of a dorsal notochord implant on formation of commissural neurons within the neural tube}

Because neurofilament immunoreactivity cannot distinguish classes of neurons, the data in the previous sections cannot reliably predict the types of neurons present in the induced floor plate region. Some appear morphologically similar to commissural neurons, which are readily identifiable morphologically by their rounded cell bodies and ventrally directed axons. This population first arises in the dorsal portion of the neural tube and sends its axons ventrally toward to the floor plate (Tessier-Lavigne et al., 1988). Commissural neurons subsequently form along the entire lateral margin of the neural tube. In order to distinguish commissural neurons from other neurons, we have used an antibody against CRABP which selectively recognizes this neuronal population within the neural tube (Maden et al., 1989).

In order to determine whether a dorsal floor plate affects neural-tube-derived structures other than the neural crest, we examined the effects of an ectopic notochord on the distribution of commissural neurons. To this end, we examined CRABP immunoreactivity in embryos receiving a notochord graft either on the dorsum or the dorsolateral por- 

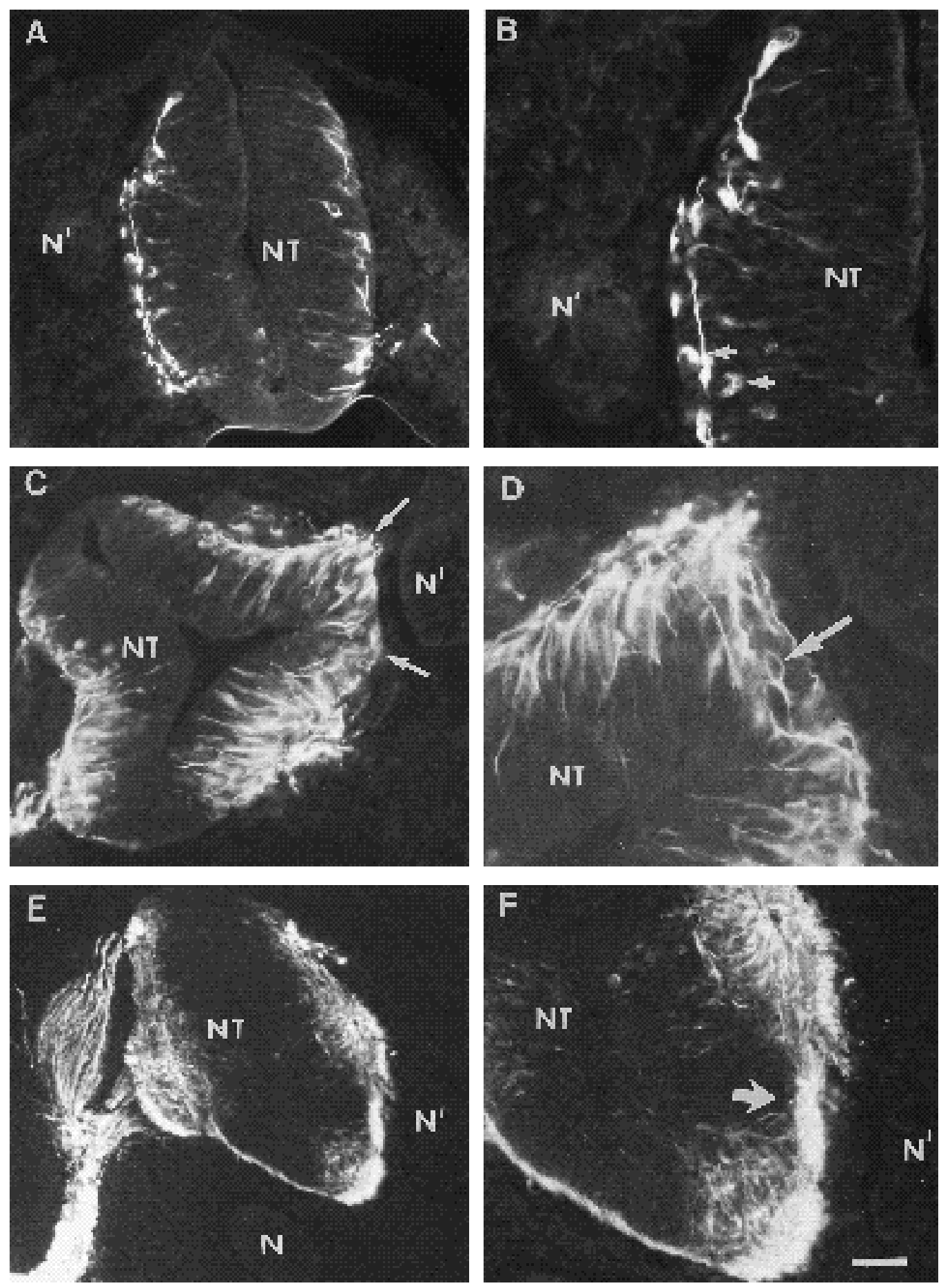

Fig. 6. Fluorescence micrographs of transverse sections through embryos receiving notochord implants at stage 10-11 and examined for neurofilament immunoreactivity at subsequent stages. (A,B) Low and high magnification micrographs, respectively of a section through a stage 16 embryo, in which numerous neurofilament immunoreactive cell bodies and processes were observed adjacent to the implanted notochord $\left(\mathrm{N}^{\prime}\right)$. (C) In a stage-19 embryo, neurofilament immunoreactivity was present in both cell bodies and axonal processes throughout the induced floor plate region adjacent to the implanted notochord; arrows indicate the borders of induced floor plate. (D) An adjacent section to $\mathrm{C}$ at higher magnification illustrating a neuron with a ventrally directed axon (arrow) reminiscent of a commissural neuron within the induced floor plate; numerous axons were seen in the floor plate region. (E, F) Adjacent sections through an embryo fixed at stage 23-24 viewed at low and high magnifications, respectively. Numerous neurofilament immunoreactive cells and axons were present in the neural tube. However, the floor plate region (arrow) was devoid of cell bodies by this stage and contained only axonal processes on its basal surface. Bar $=50 \mu \mathrm{m}$ in $\mathrm{A}, \mathrm{C} ; 15 \mu \mathrm{m}$ in $\mathrm{B}, \mathrm{F} ; 20 \mu \mathrm{m}$ in $\mathrm{D}$; and $35 \mu \mathrm{m}$ in $\mathrm{E}$. 
tion of the neural tube. Observations were made from sections through 7 embryos, examined 1.5-2 days after implantation (stages 17 through 20). Commissural neurons were defined by their CRABP immunoreactivity; also, in many of the cells, we could visualize their rounded cell bodies and ventrally projecting axons. CRABP immunoreactive neurons were first observed in embryos fixed at stage 15, when a few immunoreactive cells were seen in the lateral regions of the neural tube. The number of commissural neurons increased in embryos fixed at progressive stages. In embryos with dorsolaterally implanted notochords, distinct commissural neurons were present on both the unoperated and notochord-implanted side of the embryo. They were often found in the neural tube immediately medial to the implanted notochord, in the region of the induced floor plate (Fig. 7A,B). Even when the notochord was grafted to the dorsal aspect of the neural tube, CRABP immunoreactive neurons were observed adjacent to the implant in the induced floor plate region (Fig. 7C,D). In some embryos, fewer CRABP reactive cells were seen in the region of the ectopic notochord than on the contralateral side. This was most evident in embryos fixed at later times, consistent with the possibility that commissural neurons may be cleared with progressive developmental times. The results demonstrate that the implanted notochord does not prevent initial differentiation of commissural neurons in its vicinity, as suggested by the neurofilament immunoreactivity.

\section{Discussion}

We have examined whether interactions between the notochord and the dorsal neural tube suppress dorsal properties such as formation of neural crest cells and commissural neurons. The results demonstrate that neural crest cells emigrate from the dorsal neural tube even in the presence of a dorsal or dorsolateral notochord and floor plate. Furthermore, commissural neurons also differentiate in the induced floor plate region. Thus, the notochord cannot prevent neural crest cell formation or commissural neuron differentiation. Taken together with previous studies on ventralization of the neural tube (van Straaten et al, 1988; Yamada et al., 1991), our data suggest that the dorsal and ventral properties of the neural tube may be regulated independently. Portions of the dorsal neural tube, for example, may be programmed to form neural crest and commissural neurons in a manner that cannot be suppressed by contact with the notochord. However, the notochord does influence the subsequent development of neural crest cells and commissural neurons. It produces a perinotochordal matrix which is inhibitory for neural crest cells (Newgreen et al., 1986; Pettway et al., 1990) and it alters the position and size of neural crest-derived dorsal root ganglia.

By examining CRABP and neurofilament immunoreactivities, we have made a number of novel observations regarding the distribution of commissural neurons and other axonal processes in the region of the induced floor plate. First, we observed CRABP-reactive commissural neurons in the region of the induced floor plate, suggesting that these 'dorsal' neurons are not subverted to form ventral motor neurons. Rather, both dorsal (our findings) and ventral
(Yamada et al., 1991) neuronal types appear to co-exist in the vicinity of the induced floor plate. Second, the induced floor plate is either different or temporally delayed compared with the endogenous floor plate with regard to its content of neurons and axons. The host floor plate is a neuron-free region which only has axonal processes circumnavigating its basal side. In contrast, the induced floor plate contains abundant axonal processes as well as neuronal cell bodies one to two days after notochordal implantation. At later stages, neuronal cells bodies and processes are no longer observed in the induced floor plate, suggesting that a significant reorganization occurs. Our observations cannot distinguish between the possibility that commissural neurons in the floor plate region are removed by selective cell death or by active migration of cell bodies. However, movement of other types of neurons within the spinal cord has been documented (Markham and Vaughn, 1991). These results illustrate that secondary induction of a floor plate may not mimic the endogenous induction in all respects. Interestingly, the induced and endogenous floor plates become morphologically similar by 3 days after implantation. Some neurofilament immunoreactive cells occasionally are observed in the endogenous floor plate at early stages (unpublished observation). Thus, it is possible that clearance of neurons from the floor plate region occurs during normal development and is temporally delayed in the induced floor plate.

Signals from the notochord and floor plate play a role in establishing cell identity along the dorsoventral axis of the neural tube (van Straaten et al., 1988; Yamada et al., 1991). An ectopic notochord grafted adjacent to the neural tube appears to induce differentiation of the floor plate and motor neurons (van Straaten et al., 1988, 1989; Yamada et al., 1991). Once formed, the floor plate itself can induce the formation of additional floor plates and motor neurons (Yamada et al., 1991). Taken together, these results suggest that the notochord and floor plate can cause ventralization of neural tube structures. A possible mechanism for these findings, proposed by Yamada and colleagues (1991), is that the notochord and floor plate may be sources of an inducing substance that diffuses through the neuroepithelium. Neural tube cells may respond to the local concentration of this diffusible factor such that the cells experiencing highest concentrations become floor plate whereas cells detecting a lower concentration become motor neurons. The differentiation of dorsal cell types, such as commissural neurons within the neural tube, occurs even in the absence of the notochord (Yamada et al., 1991). Thus, some 'dorsal' properties may be constitutive or may require instructive cues from other sources. For example, they could occur via interactions between the neural plate and chordamesoderm prior to formation of the notochord or from lateral interactions within the ectoderm/neuroectoderm. The finding that some commissural neurons cannot be subverted by a notochord implanted dorsally during neural tube closure may not be surprising, since a subpopulation of these neurons is born quite early in development, during or prior to the early stages of neurulation (Sechrist and Bronner-Fraser, 1991; Yaginuma et al., 1990). In contrast, other dorsal markers, such as the AC4 epitope are displaced after a dorsolateral notochord graft (Yamada et al., 
1991). Since the antigen has yet to be identified, its relationship to neural crest cells and commissural neurons is presently unknown. Thus, implanted notochords may have differential effects on various 'dorsal' properties. Although it is clear that many commissural neurons can differentiate in the vicinity of an ectopic floor plate, we cannot rule out the possibility that a subpopulation of presumptive commissural neurons changes their prospective fate after notochord implantation to become motor neurons, as suggested by Yamada and colleagues (1991). Similar to commissural neurons, neural crest cells arise even in the presence of a dorsal notochord. The finding that both commissural neurons and neural crest cells are unaffected by the presence of a dorsal notochord is consistent with the observation that these two populations can share a common lineage (Bronner-Fraser and Fraser, 1989).

What factors are responsible for formation of the neural crest? The present results suggest that, in the trunk region, induction of the neural crest within the dorsal neural tube occurs prior to tube closure. In contrast, induction of the floor plate occurs following neural tube closure and removal of the notochord after neurulation results in the absence of the floor plate and motor neurons (Yamada et al., 1991). Therefore, the acquisition of 'dorsal' traits may occur well before acquisition of 'ventral' traits. Grafting experiments suggest that neural crest cells may form when the neural tube and ectoderm are juxtaposed. For example, transplantation of axolotl neural plate into epidermis or vice versa results in the formation of neural crest derivatives (melanocytes and spinal ganglia) at the border between the juxtaposed tissues; interestingly both epidermis and neural plate can contribute to the newly formed neural crest derivatives (Moury and Jacobson, 1990). Even after neural tube closure, the presumptive neural crest is not a segregated population; rather, a single precursor cell in the dorsal neural tube can give rise to neural crest cells, commissural neurons and/or roof plate cells (Bronner-Fraser and Fraser, 1988, 1989). Thus, dorsal neural tube cells can assume a number of 'dorsal' phenotypes. Although neural crest cells normally arise from the dorsal neural tube, they can be experimentally induced from the ventral neural tube at cranial levels. When as much as half of the midbrain and/or hindbrain is removed, the remaining ventral region of the neural tube can completely regulate the missing dorsal neural crest derivatives (Scherson et al., unpublished data). Whether a similar regulation can occur at trunk levels has yet to be tested. In light of these observations suggesting that neural crest cells can arise from the entire dorsoventral extent of the neural tube, it is interesting to note that commissural neurons also arise both dorsolaterally and ventrolaterally (Yaginuma et al., 1990).

In addition to the notochord, the neural tube itself appears to affect the polarity of the developing nervous system. When the neural tube is rotated shortly after tube closure (stage 10-12) to invert its dorsoventral orientation by $180^{\circ}$, neural crest cells emerge in ventral regions of the embryo and migrate both dorsally and ventrally (Weston, 1963; Stern et al., 1991). After dorsoventral rotation of the neural tube, the central and peripheral nervous systems form with inverted polarity with respect to the embryo, but normally with respect to the rotated neural tube. The exception is the sympathetic ganglia, which only form in their normal sites and appear to require interactions with either the ventral neural tube or notochord in order to differentiate into adrenergic cells (Stern et al., 1991). These results demonstrate that the undifferentiated neural tube has intrinsic polarity prior to the time of rotation and dictates the orientation of dorsal root ganglion formation.

The notochord appears to have a variety of functions invoked at different stages of development. During the time of neural crest cell migration, the notochord appears to inhibit neural crest cells in its immediate vicinity. In culture, neural crest cells avoid notochords (Newgreen et al., 1986). In vivo, neural crest cells avoid implanted notochords that are placed in their trajectory of migration (Pettway et al., 1990). The inhibitory substance produced by the notochord may be a chondroitin sulfate proteoglycan (Newgreen et al., 1986; Pettway et al., 1990). Several hours after the onset of neural crest migration, motor axons extend their processes out of the neural tube through the ventral root. The notochord has been shown to influence the number of ventral root fascicles (van Straaten et al., 1988, 1989; Yamada et al., 1991). Accordingly, we observed numerous ventral root axon bundles emerging both ventrally and dorsally from neural tubes in the vicinity of implanted notochords. Interestingly, the notochord not only induces formation of extra ventral roots but it also influences their projection pattern into the periphery. Tosney and Oakley (1990) have found that the notochord inhibits ventral root axons in the perinotochordal space. These axons appear to move preferentially along the border of the perinotochordal matrix.

In addition to influencing neural crest cell and axonal migration, the present results demonstrate that implanted notochords can alter the position and size of neural-crestderived dorsal root ganglia (DRG). The finding that neural crest cells, DRGs and axons are all misplaced by the notochord is consistent with the possibility that the notochord produces an inhibitory substance(s) that discourages contact with neural crest cells throughout the migratory period and during gangliogenesis. A small number of neural crest cells are able to circumnavigate the implanted notochords to form sympathetic ganglia of normal size and position. The mechanisms underlying size-reduction of the DRG are unclear; perhaps the notochord produces a substance that inhibits neural crest cell division or imposes a physical constraint on neural crest cell-cell interactions, which alters DRG cell number. Alternatively, a migration-inhibiting substance produced by the notochord may constrain the space available for ganglion formation, thereby indirectly resulting in smaller DRGs.

In conclusion, the present results demonstrate that at least some of the dorsal properties of the neural tube, its ability to form neural crest and commissural neurons, cannot be subverted by the presence of a grafted notochord. Although the notochord plays an important role in neural tube polarity, there must be an additional instructive influence that causes 'dorsalization'. This inductive signal is likely to occur quite early, prior to neural fold formation. An interesting question is whether a common signal influences the differentiation of dorsal structures such as commissural neurons, neural crest cells and perhaps other dorsal cell 
types. Because neural crest cells can share a common lineage with dorsal neural tube cells (Bronner-Fraser and Fraser, 1989), it is intriguing to speculate that their origin and induction may be interdependent.

We thank Drs Scott Fraser, Mark Selleck, John Sechrist and Zoe Pettway for helpful comments on the manuscript, Dr John Sechrist for helping with analysis of neurofilament immunoreactivity and Mary Flowers for her technical assistance. We gratefully acknowledge the generous gifts of antibodies from a number of colleagues: Drs H. Tanaka and T. Yamada for the SC1 antibody, Dr Virginia Lee for the neurofilament antibody and Drs Ulf Eriksson and Malcolm Maden for the anti-CRABP antibodies. This work was supported by USPHS HD-25138.

\section{References}

Bronner-Fraser, M. (1985). Alterations in neural crest migration by a monoclonal antibody that affects cell adhesion. J. Cell Biol. 101, 610-617.

Bronner-Fraser, M. (1986). Analysis of the early stages of trunk neural crest migration in avian embryos using the monoclonal antibody HNK-1. Dev. Biol. 115, 44-55.

Bronner-Fraser, M. and Fraser, S. (1988). Cell lineage analysis shows multipotentiality of some avian neural crest cells. Nature 335, 161-164.

Bronner-Fraser, M. and Fraser, S. (1989). Developmental potential of avian trunk neural crest cells in situ. Neuron 3, 755-766.

Eriksson, U., Hansson, E., Norlinder, H., Busch, C., Sundelin, J. and Peterson, P. A. (1987). Quantitation and tissue localisation of the cellular retinoic acid-binding protein. J. Cell Physiol. 133, 482-490.

Hamburger, V. and Hamilton, H. L. (1951). A series of normal stages in the development of the chick embryo. J. Morph. 88, 49-92.

Lallier, T. and Bronner-Fraser, M. (1988). A spatial and temporal analysis of dorsal root ganglion formation in the avian embryo. Dev. Biol. 127, 99-112.

LeDouarin, N. (1974). Cell recognition based on natural morphological nuclear markers. Med. Biol. 52, 281-319.

Lee, V., Carden, M., Schlaepfer, W. and Trojanowski, J. (1987). Monoclonal antibodies distinguish several differentially phosphorylated states of the two largest rat neurofilament subunits (NF-H and NF-M) and demonstrate their existence in the normal nervous system of adult rats. $J$. Neurosci. 7, 3474-3489.

Maden, M., Ong, D. E., Summerbell, D., Chytil, F. and Hirst, E. A. (1989). Cellular retinoic acid binding protein and the role of retinoic acid in the development of the chick embryo. Dev. Biol. 135, 124-132.

Markham, J. A. and Vaughn, J. E. (1991). Migration patterns of sympathetic preganglionic neurons in embryonic rat spinal cord. $J$. Neurobiol. 22, 811- 822.

Moury, J. D. and Jacobson, A. G.(1990). The origins of neural crest cells in the axolotl. Dev. Biol. 141, 243-253.
Newgreen, D., Scheel, M. and Kastner, V. (1986). Morphogenesis of sclerotome and neural crest in avian embryos: In vivo studies on the role of notochordal extracellular matrix. Cell Tissue Res. 244, 299-313.

Pettway, Z., Guillory, G. and Bronner-Fraser, M. (1990). Absence of neural crest cells from the region surrounding the implanted notochords in situ. Dev. Biol. 142, 335-345.

Placzek, M., Tessier-Lavigne, M., Jessell, T. and Dodd, J. (1990). Orientation of commissural axons in vitro in response to a floor plate derived chemoattractant. Dev. Biol. 110, 19-30

Rickmann, M., Fawcett, J. W. and Keynes, R. J. (1985). The migration of neural crest cells and the growth of motor axons through the rostral half of the chick somite. J. Embryol.Exp. Morph. 90, 437-455.

Sechrist, J. and Bronner-Fraser, M. (1991). Reticular neurons in the chick hindbrain are born prior to neurulation. Neuron 7, 947-963.

Smith, J. and Schoenwolf, G. C. (1989). Notochordal induction of cell wedging in the chick neural plate and its role in neural tube formation. $J$. Exp. Zool. 25, 49-62.

Stern, C. D., Artinger, K. B. and Bronner-Fraser, M. (1991). Tissue interactions affecting the migration and differentiation of neural crest cells in the chick embryo. Development 113, 207-216.

Serbedzija, G., Bronner-Fraser, M. and Fraser, S. (1989). Vital dye analysis of the timing and pathways of avian trunk neural crest cell migration. Development 106, 806-816.

Serbedzija, G., Fraser, S. E. and Bronner-Fraser, M. (1990). Pathways of trunk neural crest cell migration in the mouse embryo revealed by vital dye analysis. Development 108, 605-612.

Tessier-Lavigne, M., Placzek, M., Lumsden, A., Dodd, J. and Jessell, T. M. (1988). Chemotropic guidance of developing axons in the mammalian central nervous system. Nature 336, 775-778.

Tosney, K.W. and Oakley, R.A. (1990). The perinotochordal mesenchyme acts as a barrier to axon advance in the chick embryo: Implications for a general mechanism of axon guidance. Exp. Neurol. 109, 75-89.

van Straaten, H. W. M., Hekking, E. J. L. M., Wiertz-Hoessels, F. T. and Drukker, J. (1988). Effects of the notochord on the differentiation of a floor plate area in the neural tube of the chick embryo. Anat. Embryol. 177, 317-324.

van Straaten, H. W. M., Hekking, J. W. M., Beursgens, J. P. W. M., Terwindt-Rouwenhorst, E. and Drukker, J. (1989). Effect of the notochord on proliferation and differentiation in the neural tube of the chick embryo. Development 107, 793-803.

Yaginuma, H., Shjiga, T., Homma, S., Ishihara, R. and Oppenheim, R. (1990). Identification of early developing axon projections from spinal interneurons in the chick embryo with a neuron specific $\beta$-tubulin antibody: evidence for a new 'pioneer' pathway in the spinal cord. Development 108, 705-716.

Yamada, T., Placzek, M., Tanaka, H., Dodd, J. and Jessell, T. M. (1991). Control of cell pattern in the developing nervous system: Polarizing activity of the floor plate and notochord. Cell 64, 635-647.

Weston, J. A. (1963). A radiographic analysis of the migration and localization of trunk neural crest cells in the chick. Dev. Biol. 6, 279310.

(Accepted 9 September 1992) 
Dev 1978

Fig. 3. Transverse sections through embryos that received a small focal injection of DiI into the neural fold region prior to implantation of an extra notochord ( $\left.\mathrm{N}^{\prime}\right)$ at stages 9-11. Note that, in many cases, the implanted notochord prevented fusion of the neural folds, causing the formation of a floor plate in the presumptive roof plate region. (A) An embryo fixed at stage 15 and viewed through the fluorescein filter set (through which DiI is evident). DiI-labelled cells were observed in the dorsal neural tube, but no labelled cells have emigrated from the neural tube (NT). Occasionally, the notochordal cells also were labelled with DiI. (B) An embryo fixed at stage 16, viewed through the fluorescein filter set. Some DiI-labelled neural crest cells (arrow) have emigrated from the neural tube. (C) An embryo fixed at stage 17 , viewed through the rhodamine filter set (through which maximal DiI signal is detected). Numerous DiI-labelled neural crest cells (arrows) were observed in the sclerotome on both sides of the embryo. The ectoderm also was labelled with DiI. (D) An embryo fixed at stage 18, viewed through the rhodamine filter set. Numerous DiI-labelled neural crest cells were observed unilaterally throughout the rostral half of the sclerotome. Bar $=40 \mu \mathrm{m}$.

Fig. 7. Differential interference micrographs of CRABP immunoreactivity in embryos receiving notochord grafts at stage 10 and fixed at stage 20. (A, B) Low and high magnification micrographs of a transverse section in which the ectopic notochord ( $\left.\mathrm{N}^{\prime}\right)$, grafted lateral to the neural tube induced a wedge-shaped floor plate; the arrows indicate the borders of the induced floor plate region. Numerous CRABPpositive cells and axons, visualized by their brown reaction product, were observed within the induced floor plate region. (C, D) Low and high magnification micrographs, respectively, of a transverse section in which the notochord was implanted in the dorsal portion of the neural tube (NT). In this embryo, the dorsally located notochord displaced the dorsomedial neural tube such that it appeared to be in the dorsal midline. The cell body and axon of a commissural neuron (arrows) are clearly visible in the neural tube immediately below the ectopic notochord in a dorsomedial position, suggesting that the notochord did not prevent differentiation of commissural neurons in the dorsal neural tube. Bar $=50 \mu \mathrm{m}$ in $\mathrm{A}, \mathrm{C} ; 15 \mu \mathrm{m}$ in $\mathrm{B}, \mathrm{D}$. 